OPEN ACCESS

Edited by:

loan Opris,

Wake Forest University School of

Medicine, USA

Reviewed by:

Krystal Lynn Parker,

University of lowa, USA

Puiu F. Balan,

KU Leuven, Belgium

*Correspondence:

Katharina M. Kubera,

Center for Psychosocial Medicine,

Department of General Psychiatry,

University of Heidelberg, Voßstraße 4, 69115 Heidelberg, Germany katharina.kubera@med.uniheidelberg.de

Received: 03 March 2015 Accepted: 07 September 2015

Published: 12 October 2015

Citation:

Kubera KM, Barth A, Hirjak D, Thomann PA and Wolf RC (2015) Noninvasive brain stimulation for the treatment of auditory verbal hallucinations in schizophrenia methods, effects and challenges.

Front. Syst. Neurosci. 9:131. doi: 10.3389/fnsys.2015.00131

\section{Noninvasive brain stimulation for the treatment of auditory verbal hallucinations in schizophrenia: methods, effects and challenges}

\author{
Katharina M. Kubera ${ }^{1 *}$, Anja Barth ${ }^{1}$, Dusan Hirjak ${ }^{1}$, Philipp A. Thomann ${ }^{1}$ \\ and Robert C. Wolf ${ }^{1,2}$ \\ ${ }^{1}$ Center for Psychosocial Medicine, Department of General Psychiatry, University of Heidelberg, Heidelberg, Germany, \\ ${ }^{2}$ Department of Psychiatry, Psychotherapy and Psychosomatics, Saarland University, Homburg, Germany
}

This mini-review focuses on noninvasive brain stimulation techniques as an augmentation method for the treatment of persistent auditory verbal hallucinations (AVH) in patients with schizophrenia. Paradigmatically, we place emphasis on transcranial magnetic stimulation (TMS). We specifically discuss rationales of stimulation and consider methodological questions together with issues of phenotypic diversity in individuals with drug-refractory and persistent $\mathrm{AVH}$. Eventually, we provide a brief outlook for future investigations and treatment directions. Taken together, current evidence suggests TMS as a promising method in the treatment of $\mathrm{AVH}$. Low-frequency stimulation of the superior temporal cortex (STC) may reduce symptom severity and frequency. Yet clinical effects are of relatively short duration and effect sizes appear to decrease over time along with publication of larger trials. Apart from considering other innovative stimulation techniques, such as transcranial Direct Current Stimulation (tDCS), and optimizing stimulation protocols, treatment of AVH using noninvasive brain stimulation will essentially rely on accurate identification of potential responders and non-responders for these treatment modalities. In this regard, future studies will need to consider distinct phenotypic presentations of $\mathrm{AVH}$ in patients with schizophrenia, together with the putative functional neurocircuitry underlying these phenotypes.

Keywords: transcranial magnetic stimulation, auditory verbal hallucinations, phenotypes, schizophrenia, brain stimulation, brain function

\section{Introduction}

Auditory verbal hallucinations (AVH) are defined as auditive perceptions involving a verbal aspect in the absence of a provoking external stimulus (Aleman and de Haan, 1998). They represent a core symptom of schizophrenia and related spectrum disorders, but they also frequently occur in other psychiatric entities and in the non-psychiatric general population. In schizophrenia the term AVH comprises a multi-dimensional and heterogeneous group of symptoms that can be differentiated by certain phenomenological aspects such as subjective loudness, acoustic clarity, location and subjective reality. About $60-80 \%$ of patients affected by schizophrenia experience AVH (Aleman and de Haan, 1998; Hugdahl et al., 2008), such as conversing, commenting or imperative spoken speech in distinct voices. These symptoms, especially when the verbal content is experienced as negative, intrusive or persecutory, 
often induce high levels of distress and lead to significant psychosocial impairment. AVH are a highly relevant feature of schizophrenia that have attracted extensive clinical, phenomenological and neurobiological interest, yet treating these symptoms, especially in persons suffering from persistent AVH which do either not or not sufficiently respond to psychopharmacotherapy, is still a major clinical challenge. In approximately $25 \%$ of patients with schizophrenia, AVH are refractory to psychotropic drug treatment and can chronically persist (Shergill et al., 1998). Currently, there are no randomized controlled trials available which specifically investigated effects of psychopharmacotherapy (either monotherapy or combined drug regimes) on AVH severity reduction or full symptom remission. In a recent review (Sommer et al., 2012), data from the European First-Episode Schizophrenia Trial (EUFEST) was used to assess effects of five antipsychotic agents on $\mathrm{AVH}$ severity. Superiority of one treatment option against another was not confirmed for AVH severity (Sommer et al., 2012). Clinically, clozapine is still the drug of choice for patients with AVH who are resistant to two other antipsychotic agents. At present, no clinical trial has been published that specifically compares the efficacy of clozapine in comparison to other antipsychotic agents in the treatment of drug-resistant AVH.

\section{Transcranial Magnetic Stimulation}

Given the need for effective treatment modalities, it is not surprising to see that over the past decade brain stimulation techniques have been increasingly used to ameliorate symptom burden in patients with schizophrenia suffering from persistent and mostly pharmacorefractory AVH. Among these approaches, a possible augmentation strategy for the treatment of psychiatric disorders (in particular catatonia and severe depression) is elctroconvulsive therapy (ECT). For patients with schizophrenia a meta-analysis of 10 double-blind RCT showed a significant effect for ECT (Tharyan and Adams, 2005), although none of the studies provided any specific details on AVH improvement (Sommer et al., 2012). In a recent review Nieuwdorp and colleagues summarized different stimulation methods including transcranial magnetic stimulation (TMS), ECT and transcranial Direct Current Stimulation (tDCS) in patients with medication-resistant psychosis (Nieuwdorp et al., 2015). The authors concluded that currently there is only weak evidence for stimulation techniques to relieve pharmacorefractory psychosis. Specifically considering AVH, further studies are needed to draw any strong conclusions about ECT as a treatment option for patients presenting with persistent AVH.

In the last decade, TMS has evolved into a therapeutic modality for several psychiatric and neurological symptoms. In particular, TMS is widely used to treat patients with major depression, obsessive-compulsive disorder (OCD) and specific symptoms of schizophrenia (AVH and negative symptoms) (Slotema et al., 2010). Its application as an adjunctive therapy is currently proposed by European specialists with evidence level C (Lefaucheur et al., 2014) taking into account that it is generally regarded as safe. We consider the application of TMS for treating individuals presenting with persistent $\mathrm{AVH}$ as paradigmatic. The use of TMS impressively illustrates a translational approach from basic neuroscience/neuroimaging to clinical treatment. However, it also illustrates fundamental methodological, neurobiological and phenomenological questions and challenges, which we will refer to in the following paragraphs.

\section{TMS: Putative Mechanisms of Action}

TMS is a technique which allows a non-invasive stimulation of cortical neurons through the scalp. Originally, TMS was implemented as a neurophysiological tool for the study of the human motor system (Barker et al., 1985). Put simply, TMS uses a strong pulse of electrical current in a coil which is placed over the brain generating rapidly pulsating magnetic fields, which pass through the scalp, skull, and meninges, into the brain (Wassermann and Zimmermann, 2012). Thus, changing magnetic fields produce electrical impulses that stimulate superficial cortical neurons $2-3 \mathrm{~cm}$ below the device (Wassermann and Zimmermann, 2012). Modern devices can generate a rapid succession of pulses, called repetitive TMS (rTMS) by producing a relatively powerful magnetic field (about 1.5-3T), but only lasting very shortly (ms) (George and Aston-Jones, 2010). Frequencies of $1 \mathrm{~Hz}$ or lower are considered to be inhibitory, while frequencies of $5 \mathrm{~Hz}$ and higher are considered to be excitatory (Aleman, 2013). The specific topology of the induced electrical field in the brain is a source of uncertainty, because it is influenced by the complex shape and diverse conductivity of the cranial contents (Wassermann and Zimmermann, 2012), e.g., cerebrospinal fluid and foraminas in cranial bone. Long-term potentiation (LTP) and long-term depression (LTD) are believed to be key processes underlying long-term effects of rTMS (Chervyakov et al., 2015). In vitro experiments of hippocampal slice cultures suggest that rTMS can alter cortical excitability in terms of LTP of synaptic transmission inducing an increase in synaptic strength and postsynaptic AMPA receptor changes (Vlachos et al., 2012). At the level of functional connectivity, longlasting enhancement is reflected by increased hippocampalcortical network coupling after rTMS (Wang and Voss, 2015).

\section{How Effective is rTMS in the Treatment of AVH?}

As a target region for rTMS in patients with AVH the superior temporal cortex (STC) is of special interest given converging multimodal imaging evidence suggesting a crucial role in AVH generation and perception (Allen et al., 2008; Waters et al., 2012). The rationale for stimulating this region is to inhibit cortical overactivity and potentially influence generative phenomena (i.e., AVH) which are thought to be closely associated with regionally increased cortical activity. Up to now, several randomized sham-controlled studies targeting the left temporoparietal cortex have been conducted and summarized in seven meta-analyses revealing effect sizes (Hedges' "g") ranging from 0.42 (i.e., a close to moderate effect) to 1.04 (regarded as high effect; Aleman et al., 2007; Tranulis et al., 2008; Freitas et al., 2009; Slotema et al., 2012, 2014). With the inclusion of the studies with larger patient samples, the mean 
weighted effect size of rTMS directed at the left temporoparietal area for AVH appears to decrease over time, although the effect is still significant (Slotema et al., 2012, 2014; Hoffman et al., 2013). Of note, Slotema and colleagues showed that the effect of rTMS was no longer significant at one month of follow-up revealing a mean weighted effect size of 0.40 (95\% confidence interval $=-0.23-0.102$; Slotema et al., 2012). For a detailed description of the included studies, please see tables provided by Slotema and colleagues. Side effects were mild and the number of dropouts in the real TMS group was not significantly higher than in the sham group. Only few MRI studies investigated other regions than the left temporoparietal area as target regions for rTMS. Abnormal activation of the right hemisphere regions such as the inferior frontal gyrus and the postcentral gyrus is a frequently reported finding in patients who experience persistent AVH (Kuhn and Gallinat, 2012). Activation changes have been most consistently shown for areas of the prefrontal and temporal cortices (Allen et al., 2007; Sommer et al., 2008; Raij et al., 2009). Based on former findings in neuroimaging studies that both the right and the left temporal activation are associated with AVH (Shergill et al., 2000; Sommer et al., 2007) three studies directed rTMS at the right comparing with the left temporoparietal gyrus for the treatment of AVH (Lee et al., 2005; Jandl et al., 2006; Loo et al., 2010). According to these studies, no superior effects of right-sided stimulation (Slotema et al., 2014) were observed. Correspondingly, neither stimulation of Brocaś area nor its contralateral homologue was an effective target (SchonfeldtLecuona et al., 2004). Overall, these findings support the notion that deficient generation, monitoring and perception of inner speech rather than speech expression are disrupted functions in patients with persistent AVH (Shergill et al., 2000; Wolf et al., 2011). Abnormal STC function clearly plays a critical role in the expression of $\mathrm{AVH}$, especially in those patients presenting with chronic and treatment-refractory symptoms. Recent studies showed that stimulation of this region with low-frequency rTMS may reduce the severity and frequency of $\mathrm{AVH}$ in schizophrenia patients, but the duration of the effect of rTMS may be less than one month (Slotema et al., 2012).

\section{Methodological Issues with TMS and the Challenge of Treating Phenotypic Diversity}

As briefly discussed in the previous paragraph, it is noteworthy that therapeutic effects of rTMS in AVH patients are not long-lasting, and that along with publication of studies with larger patient populations, the effect size of rTMS over the left temporoparietal area has decreased over time (Slotema et al., 2012, 2014). Several studies published between 2004 and 2014 did not observe beneficial effects of rTMS in the treatment of persistent AVH (Schonfeldt-Lecuona et al., 2004; Slotema et al., 2011; Blumberger et al., 2012; Rosenberg et al., 2012; Bais et al., 2014). Several reasons may account for these phenomena. Two specific aspects of stimulation will be discussed, which may be superior to left sided STC intervention and which may also account for these variable results. Subsequently we will address the problem of the phenotypic diversity which is inherent to $\mathrm{AVH}$ both at the neural and phenomenological level.

\section{Is Bilateral Stimulation Superior?}

It may be conceivable that bilateral could be superior over unilateral stimulation, especially given known dissociations of left- vs. right-hemispheric function. Up to now, however, only one study examined bilateral rTMS of the TPJ (Bais et al., 2014). The authors suggested that AVH frequency might be one of the most sufficient parameters to measure the responsiveness of left sided rTMS. In comparison, right-sided rTMS allows for a more complete management of AVH in terms of emotional and non-linguistic aspects which are suggested to originate in the right hemisphere. Contrary to their prediction, however, Bais and could not show any beneficial effect of bilateral rTMS in comparison to left sided rTMS and sham in improving AVH (Bais et al., 2014). Neurophysiological aspects such as transcallosal inhibition, and fewer rTMS impulses (50\%) in a bilateral design (Thiel et al., 2006; Bais et al., 2014) might account for these negative results.

\section{Is STC stimulation alone sufficient?}

The functional dominance of STC stimulation over other brain regions has been questioned by accumulating neuroimaging data acquired in patients with AVH. For instance, an association between AVH-severity and STC gray matter volume loss has been suggested by univariate voxel-based morphometry studies (Modinos et al., 2013). In contrast, using a multivariate statistical approach for structural data analysis, two distinct abnormal structural networks were recently identified in patients with persistent $\mathrm{AVH}$, including a bilateral prefrontal system and a bilateral temporal/medial frontal network (Kubera et al., 2014). The latter structural network also differed between patients with persistent AVH compared to non-hallucinating patients (Kubera et al., 2014). It is possible that unilateral temporoparietal stimulation might not be sufficient to induce a relevant neuronal change in both networks, whose mutual interplay has still to be determined. Also, the relationship between structure and function still remains unresolved, e.g., in individuals with persistent AVH the impact of neural loss to neural network transmission, including effects in more remote neural networks, is unclear.

From a functional point of view, both "symptom capture" (i.e., inferring AVH-related brain activity from symptom occurrence) and "symptom interference" (i.e., inferring AVHrelated brain dysfunction from paradigm-driven data) MRI studies have been conducted to investigate neural activation patterns in schizophrenia patients experiencing treatmentresistant AVH (Lawrie et al., 2002; Mechelli et al., 2007; Wolf et al., 2011). The vast majority of these studies focused on brain activity in speech-related pathways (Lavigne et al., 2015), according to the prevailing model of AVH suggesting a link between symptom generation and dysfunctional inner speech perception and monitoring (Hugdahl et al., 2008). From these studies, the left STC emerged as regions linked to $\mathrm{AVH}$ and in turn set the rationale for targeted stimulation. 
Yet the left temporal cortex, although a crucial neural node for hallucinatory symptom expression, is not the sole region which is thought to be involved in AVH generation and persistence. The prefrontal cortices have been frequently found to exhibit abnormal neural activity in patients with $\mathrm{AVH}$, both at the level of regional function and at the level of functional connectivity (Kuhn and Gallinat, 2012; Alderson-Day et al., 2015). Although the processes subserved by abnormal prefrontal activity in patients experiencing AVH are not fully elucidated at present, several explanations have been put forward, such as deficient attentional and executive control over speech- and self-monitoring relevant brain regions. In addition, converging evidence suggests that $\mathrm{AVH}$ are not related to regional brain dysfunction alone, but rather to abnormal neural network coupling in several distinct neural networks including systems engaged in language, attention, executive function, memory and self-referential processing (Stephane et al., 2001; Allen et al., 2008; Wolf et al., 2011; Diederen et al., 2013). Thus, singlesite stimulation may not fully cover all key regions involved in AVH pathophysiology. In this regard, bilateral or bifocal stimulation could be a promising approach. Based on the hypothesis of temporal hyperactivity and frontal hypoactivation in schizophrenia patients presenting with AVH, Brunelin and colleagues used a different non-invasive stimulation method, i.e., tDCS (Brunelin et al., 2012). Unlike TMS, in tDCS a weak direct current passes through the brain between two electrodes, i.e., modulation of two spatially remote regions is possible. Brunelin and co-workers used cathodal left temporoparietal junction (TPJ) stimulation and anodal left dorsolateral prefrontal stimulation. After five days of treatment a significant decrease of hallucinatory symptoms was shown, and this effect remained significant three months after stimulation. These findings were recently replicated (Mondino et al., 2015) and provides a promising outlook for further clinical trials. Nevertheless, given that tDCS is a relatively new technique employed in $\mathrm{AVH}$ treatment, several stimulation parameters (e.g., electrode placement and stimulation intensity, frequency and duration) have to be investigated in more detail to optimize future treatment options (Koops et al., 2015).

It is noteworthy that although the lateral prefrontal and temporal cortices clearly are involved in $\mathrm{AVH}$ generation, there is also good evidence suggesting a role of cortical midline regions in AVH symptom expression. Abnormal cerebral blood flow could also be detected not only in the primary temporal cortex and Broca's area, but also in the cingulate cortex (Wolf et al., 2012; Kindler et al., 2013). In a recent study exploring resting-state functional connectivity of the brain, cross-network abnormalities could be detected between the so called "default mode network" (DMN) and the "salience network," including core midline regions such as the bilateral paracingulate cortex and bilateral anterior cingulate cortex (Alonso-Solís et al., 2015). Of note, DMN subsystems have been essentially involved in self-referential and mnemonic processes (Andrews-Hanna et al., 2010; Sambataro et al., 2013). Abnormal network interactions between the DMN and language-processing and auditory networks could well explain deficient self-monitoring and a lack of self-referential attribution of voices (Northoff and Qin,
2011). This body of evidence indicates important contributions of cortical midline regions to the pathomechanisms of persistent AVH. TMS alone might be insufficient to stimulate these regions in treatment-resistant patients.

\section{The Challenge of Phenotypic Diversity}

When treating AVH in patients with schizophrenia using focal stimulation techniques, the clinical endpoint appears to be clearly defined. In the vast majority of cases, this is at least a reduction in overall AVH severity. Yet it should be kept in mind that schizophrenia is a phenomenologically heterogeneous disorder with several distinct phenotypic presentations at both the clinical and neurobiological level, and the very same heterogeneity also applies to persons with chronic AVH. In addition, the multidimensionality of AVH has been long acknowledged by phenomenological research (Kronmüller et al., 2011; McCarthyJones et al., 2014), but research has only recently begun to specifically explore therapeutic effects on distinct symptom domains (Leff et al., 2013).

Apart from refining and technically developing stimulation techniques per se, a major focus of future research will be the identification of markers which can predict stimulation treatment response. An approach which might prove to be helpful for predicting responders and non-responders in the future is subtyping AVH patients according to both neurobiological and clinical criteria. For instance, it has been attempted, albeit with limited success, to improve responsiveness to rTMS by targeting the site of maximal neural activation associated with the hallucinatory event (Slotema et al., 2011). More recently, Homan and colleagues (Homan et al., 2012) showed that higher resting-brain perfusion as measured with arterial spin labeling in the left STC prior to treatment predicted a clinical response to rTMS (Homan et al., 2012). This marker may guide stratification strategies in future interventional trials. Also, it is important to acknowledge that certain symptom characteristics, such as location of voices in inner our outer space, may map to distinct neural correlates. In this respect, a relationship between white matter volume in the right temporal junction and spatial features such as outer vs. inner location of voices has been identified (Plaze et al., 2011). However, stimulation of the right temporal lobe could not show a superior treatment effect. A possible explanation is that there may well be structural differences between hallucinating characterized by "physical" features with yet unknown consequences for brain function and treatment response. Another explanation for nonresponse to stimulation could include neural ceiling effects, e.g., related to various degrees of subjective symptom control. Over time, patients with persistent AVH seek for ways of coping with their voices, e.g., by deliberately directing their attentional focus to specific external stimuli (which can be auditory) or by employing individual modes of verbal control. The degree of control over AVH is associated with distinct frontotemporal cortical correlates in contrast to physical or affective symptom dimensions (Wolf et al., 2012). Also, increased frontotemporal connectivity in hallucinating patients is modulated by the degree of control over verbal material (Lavigne et al., 2015). Thus, it is possible that different degrees of control over AVH severity 
prior to therapeutic stimulation could influence treatment response.

McCarthy-Jones and colleagues proposed different subtypes of AVH, which might respond to different treatment modalities. These subtypes may be identified at the levels phenomenology, cognition, neurology, etiology, treatment response, diagnosis, and voice hearer's own interpretation (McCarthy-Jones et al., 2014). Particularly, an AVH subtype characterized at a neural level by chronic deafferentiation of the auditory cortex is proposed, according to the hypothesis of AVH as misattributed forms of inner speech (Ford and Mathalon, 2005). This subtype might be specifically responsive to focal stimulation treatment, i.e., rTMS or tDCS. Furthermore, specific subtypes might show both common and distinct regions of activation in both "symptom capture" and "symptom interference" studies, so that future neuroimaging studies may consider specific subtypes in their protocol and report details of AVH phenomenology. The majority of functional neuroimaging studies used total severity and frequency scores of hallucinations (Auditory Hallucinations Rating Scale (AHRS), Auditory Hallucinations Subscale/Psychotic Symptom Rating Scale [AHS/PSYRATS]) as main outcome parameters. To discriminate more fine-grained aspects of change in hallucinations, especially in homogenous subgroups, it might be advantageous to describe different phenomenological dimensions before and their changes after focal therapy. The PSYRATS and a 4-dimensional model within the AHS has previous been recommended to integrate into research and clinical applications (Woodward et al., 2014).

\section{References}

Alderson-Day, B., McCarthy-Jones, S., and Fernyhough, C. (2015). Hearing voices in the resting brain: A review of intrinsic functional connectivity research on auditory verbal hallucinations. Neurosci. Biobehav. Rev. 55, 78-87. doi: 10. 1016/j.neubiorev.2015.04.016

Aleman, A. (2013). Use of repetitive transcranial magnetic stimulation for treatment in psychiatry. Clin. Psychopharmacol. Neurosci. 11, 53-59. doi: 10. 9758/cpn.2013.11.2.53

Aleman, A., and de Haan, E. H. (1998). On redefining hallucination. Am. J. Orthopsychiatry. 68, 656-659. doi: 10.1037/h0080376

Aleman, A., Sommer, I. E., and Kahn, R. S. (2007). Efficacy of slow repetitive transcranial magnetic stimulation in the treatment of resistant auditory hallucinations in schizophrenia: a meta-analysis. J. Clin. Psychiatry. 68, 416-421. doi: 10.4088/jcp.v68n0310

Allen, P., Aleman, A., and McGuire, P. K. (2007). Inner speech models of auditory verbal hallucinations: evidence from behavioural and neuroimaging studies. Int. Rev. Psychiatry. 19, 407-415. doi: 10.1080/09540260701486498

Allen, P., Larøi, F., McGuire, P. K., and Aleman, A. (2008). The hallucinating brain: a review of structural and functional neuroimaging studies of hallucinations. Neurosci. Biobehav. Rev. 32, 175-191. doi: 10.1016/j.neubiorev.2007.07.012

Alonso-Solís, A., Vives-Gilabert, Y., Grasa, E., Portella, M. J., Rabella, M., Sauras, R. B., et al. (2015). Resting-state functional connectivity alterations in the default network of schizophrenia patients with persistent auditory verbal hallucinations. Schizophr. Res. 161, 261-268. doi: 10.1016/j.schres.2014. 10.047

Andrews-Hanna, J. R., Reidler, J. S., Sepulcre, J., Poulin, R., and Buckner, R. L. (2010). Functional-anatomic fractionation of the brain's default network. Neuron. 65, 550-562. doi: 10.1016/j.neuron.2010.02.005

Bais, L., Vercammen, A., Stewart, R., van Es, F., Visser, B., Aleman, A., et al. (2014). Short and long term effects of left and bilateral repetitive transcranial magnetic

\section{Conclusion}

In the past decade non-invasive brain stimulation techniques became increasingly relevant for the treatment of drug-refractory AVH. Evidence for ECT for specifically treating AVH is very limited. There is evidence for beneficial effects of rTMS over left temporal and temporoparietal areas, but effect sizes for this treatment modality are moderate, and beneficial longterm effects are unlikely. It has been suggested that rTMS may reduce aberrant internally generated activity associated with AVH at the site of stimulation. Still, the role of rTMS in changing aberrant network function putatively involved in the generation of AVH has to be clarified. A further major challenge for future research is identifying of patients who do respond to treatment and those who do not or only insufficient. Supported by neuroimaging evidence, the magnitude of left STC activity has been promoted as a potential predictor of clinical improvement. Given the phenomenological diversity of schizophrenia and $\mathrm{AVH}$ in particular, it is expected that subtyping patients with AVH will essentially contribute to identify responders from non-responders for focal augmentative therapies. In this respect, in accordance with other authors we strongly advocate further development of reliable and valid psychometric assessments and neurobiological markers paralleling the optimization of future stimulation protocols. Other neuromodulatory interventions, such as tDCS provide very promising data as well but larger trials are needed.

stimulation in schizophrenia patients with auditory verbal hallucinations: a randomized controlled trial. PLoS One. 9:e108828. doi: 10.1371/journal.pone. 0108828

Barker, A. T., Jalinous, R., and Freeston, I. L. (1985). Non-invasive magnetic stimulation of human motor cortex. Lancet 1, 1106-1107. doi: 10.1016/S01406736(85)92413-4

Blumberger, D. M., Christensen, B. K., Zipursky, R. B., Moller, B., Chen, R., Fitzgerald, P. B., et al. (2012). MRI-targeted repetitive transcranial magnetic stimulation of Heschl's gyrus for refractory auditory hallucinations. Brain Stimul. 5, 577-585. doi: 10.1016/j.brs.2011.12.002

Brunelin, J., Mondino, M., Gassab, L., Haesebaert, F., Gaha, L., Suaud-Chagny, M. F., et al. (2012). Examining transcranial direct-current stimulation (tDCS) as a treatment for hallucinations in schizophrenia. Am. J. Psychiatry. 169, 719-724. doi: 10.1176/appi.ajp.2012.11071091

Chervyakov, A. V., Chernyavsky, A. Y., Sinitsyn, D. O., and Piradov, M. A. (2015) Possible Mechanisms Underlying the Therapeutic Effects of Transcranial Magnetic Stimulation. Front. Hum. Neurosci. 9:303. doi: 10.3389/fnhum.2015. 00303

Diederen, K. M., Neggers, S. F., de Weijer, A. D., van Lutterveld, R., Daalman, K., Eickhoff, S. B., et al. (2013). Aberrant resting-state connectivity in non-psychotic individuals with auditory hallucinations. Psychol. Med. 43, 1685-1696. doi: 10.1017/s0033291712002541

Ford, J. M., and Mathalon, D. H. (2005). Corollary discharge dysfunction in schizophrenia: can it explain auditory hallucinations? Int. J. Psychophysiol. 58, 179-189. doi: 10.1016/j.ijpsycho.2005.01.014

Freitas, C., Fregni, F., and Pascual-Leone, A. (2009). Meta-analysis of the effects of repetitive transcranial magnetic stimulation (rTMS) on negative and positive symptoms in schizophrenia. Schizophrenia Research. 108, 11-24. doi: 10.1016/ j.schres.2008.11.027

George, M. S., and Aston-Jones, G. (2010). Noninvasive techniques for probing neurocircuitry and treating illness: vagus nerve stimulation (VNS), transcranial 
magnetic stimulation (TMS) and transcranial direct current stimulation (tDCS). Neuropsychopharmacology. 35, 301-316. doi: 10.1038/npp.2009.87

Hoffman, R. E., Wu, K., Pittman, B., Cahill, J. D., Hawkins, K. A., Fernandez, T., et al. (2013). Transcranial magnetic stimulation of Wernicke's and Right homologous sites to curtail "voices": a randomized trial. Biol. Psychiatry. 73, 1008-1014. doi: 10.1016/j.biopsych.2013.01.016

Homan, P., Kindler, J., Hauf, M., Hubl, D., and Dierks, T. (2012). Cerebral blood flow identifies responders to transcranial magnetic stimulation in auditory verbal hallucinations. Transl. Psychiatry. 2:e189. doi: 10.1038/tp.2012.114

Hugdahl, K., Løberg, E. M., Specht, K., Steen, V. M., van Wageningen, H., and Jørgensen, H. A. (2008). Auditory hallucinations in schizophrenia: the role of cognitive, brain structural and genetic disturbances in the left temporal lobe. Front. Hum. Neurosci. 1:6. doi: 10.3389/neuro.09.006.2007

Jandl, M., Steyer, J., Weber, M., Linden, D. E., Rothmeier, J., Maurer, K., et al. (2006). Treating auditory hallucinations by transcranial magnetic stimulation: a randomized controlled cross-over trial. Neuropsychobiology. 53, 63-69. doi: $10.1159 / 000091721$

Kindler, J., Homan, P., Jann, K., Federspiel, A., Flury, R., Hauf, M., et al. (2013). Reduced neuronal activity in language-related regions after transcranial magnetic stimulation therapy for auditory verbal hallucinations. Biological Psychiatry. 73, 518-524. doi: 10.1016/j.biopsych.2012.06.019

Koops, S., van den Brink, H., and Sommer, I. E. (2015). Transcranial direct current stimulation as a treatment for auditory hallucinations. Front. Psychol. 6:244. doi: 10.3389/fpsyg.2015.00244

Kronmüller, K. T., von Bock, A., Grupe, S., Büche, L., Gentner, N. C., Rückl, S., et al. (2011). Psychometric evaluation of the Psychotic Symptom Rating Scales. Compr. Psychiatry. 52, 102-108. doi: 10.1016/j.comppsych.2010.04.014

Kubera, K. M., Sambataro, F., Vasic, N., Wolf, N. D., Frasch, K., Hirjak, D., et al. (2014). Source-based morphometry of gray matter volume in patients with schizophrenia who have persistent auditory verbal hallucinations. Prog. Neuropsychopharmacol. Biol. Psychiatry. 50, 102-109. doi: 10.1016/j.pnpbp. 2013.11.015

Kuhn, S., and Gallinat, J. (2012). Quantitative meta-analysis on state and trait aspects of auditory verbal hallucinations in schizophrenia. Schizophr. Bull. 38, 779-786. doi: 10.1093/schbul/sbq152

Lavigne, K. M., Rapin, L. A., Metzak, P. D., Whitman, J. C., Jung, K., Dohen, M., et al. (2015). Left-dominant temporal-frontal hypercoupling in schizophrenia patients with hallucinations during speech perception. Schizophr. Bull. 41, 259-567. doi: 10.1093/schbul/sbu004

Lawrie, S. M., Buechel, C., Whalley, H. C., Frith, C. D., Friston, K. J., and Johnstone, E. C. (2002). Reduced frontotemporal functional connectivity in schizophrenia associated with auditory hallucinations. Biol. Psychiatry. 51, 1008-1011. doi: 10.1016/s0006-3223(02)01316-1

Lee, S. H., Kim, W., Chung, Y. C., Jung, K. H., Bahk, W. M., Jun, T. Y., et al. (2005). A double blind study showing that two weeks of daily repetitive TMS over the left or right temporoparietal cortex reduces symptoms in patients with schizophrenia who are having treatment-refractory auditory hallucinations. Neurosci. Lett. 376, 177-181. doi: 10.1016/j.neulet.2004.11.048

Lefaucheur, J. P., Andre-Obadia, N., Antal, A., Ayache, S. S., Baeken, C., Benninger, D. H., et al. (2014). Evidence-based guidelines on the therapeutic use of repetitive transcranial magnetic stimulation (rTMS). Clin. Neurophysiol. 125, 2150-2206. doi: 10.1016/j.clinph.2014.05.021

Leff, J., Williams, G., Huckvale, M. A., Arbuthnot, M., and Leff, A. P. (2013). Computer-assisted therapy for medication-resistant auditory hallucinations: proof-of-concept study. Br. J. Psychiatry. 202, 428-433. doi: 10.1192/bjp.bp. 112.124883

Loo, C. K., Sainsbury, K., Mitchell, P., Hadzi-Pavlovic, D., and Sachdev, P. S. (2010). A sham-controlled trial of left and right temporal rTMS for the treatment of auditory hallucinations. Psychol. Med. 40, 541-546. doi: 10. $1017 / \mathrm{s} 0033291709990900$

McCarthy-Jones, S., Thomas, N., Strauss, C., Dodgson, G., Jones, N., Woods, A., et al. (2014). Better than mermaids and stray dogs? Subtyping auditory verbal hallucinations and its implications for research and practice. Schizophr. Bull. 4, S275-S284. doi: 10.1093/schbul/sbu018

Mechelli, A., Allen, P., Amaro, E., Jr., Fu, C. H., Williams, S. C., Brammer, M. J., et al. (2007). Misattribution of speech and impaired connectivity in patients with auditory verbal hallucinations. Hum. Brain Mapp. 28, 1213-1222. doi: 10. $1002 / \mathrm{hbm} .20341$
Modinos, G., Costafreda, S. G., van Tol, M. J., McGuire, P. K., Aleman, A., and Allen, P. (2013). Neuroanatomy of auditory verbal hallucinations in schizophrenia: a quantitative meta-analysis of voxelbased morphometry studies. Cortex. 49, 1046-1055. doi: 10.1016/j.cortex.2012. 01.009

Mondino, M., Haesebaert, F., Poulet, E., Suaud-Chagny, M. F., and Brunelin, J. (2015). Fronto-temporal transcranial Direct Current Stimulation (tDCS) reduces source-monitoring deficits and auditory hallucinations in patients with schizophrenia. Schizophr. Res. 161, 515-516. doi: 10.1016/j.schres.2014. 10.054

Nieuwdorp, W., Koops, S., Somers, M., and Sommer, I. E. (2015). Transcranial magnetic stimulation, transcranial direct current stimulation and electroconvulsive therapy for medication-resistant psychosis of schizophrenia. Curr. Opin. Psychiatry. 28, 222-228. doi: 10.1097/yco.0000000000000156

Northoff, G., and Qin, P. (2011). How can the brain's resting state activity generate hallucinations? A 'resting state hypothesis' of auditory verbal hallucinations. Schizophr. Res. 127, 202-214. doi: 10.1016/j.schres.2010.11.009

Plaze, M., Paillere-Martinot, M. L., Penttila, J., Januel, D., de Beaurepaire, R., Bellivier, F., et al. (2011). "Where do auditory hallucinations come from?"- a brain morphometry study of schizophrenia patients with inner or outer space hallucinations. Schizophr. Bull. 37, 212-221. doi: 10.1093/schbul/sbp081

Raij, T. T., Valkonen-Korhonen, M., Holi, M., Therman, S., Lehtonen, J., and Hari, R. (2009). Reality of auditory verbal hallucinations. Brain. 132, 2994-3001. doi: 10.1093/brain/awp186

Rosenberg, O., Gersner, R., Klein, L. D., Kotler, M., Zangen, A., and Dannon, P. (2012). Deep transcranial magnetic stimulation add-on for the treatment of auditory hallucinations: a double-blind study. Ann. Gen. Psychiatry. 11:13. doi: $10.1186 / 1744-859 \mathrm{x}-11-13$

Sambataro, F., Wolf, N. D., Pennuto, M., Vasic, N., and Wolf, R. C. (2013). Revisiting default mode network function in major depression: evidence for disrupted subsystem connectivity. Psychol. Med. 44, 2041-2051. doi: 10. $1017 /$ s0033291713002596

Schonfeldt-Lecuona, C., Grön, G., Walter, H., Büchler, N., Wunderlich, A., Spitzer, M., et al. (2004). Stereotaxic rTMS for the treatment of auditory hallucinations in schizophrenia. Neuroreport. 15, 1669-1673. doi: 10.1097/01. wnr.0000126504.89983.ec

Shergill, S. S., Bullmore, E., Simmons, A., Murray, R., and McGuire, P. (2000). Functional anatomy of auditory verbal imagery in schizophrenic patients with auditory hallucinations. Am. J. Psychiatry. 157, 1691-1693. doi: 10.1176/appi. ajp.157.10.1691

Shergill, S. S., Murray, R. M., and McGuire, P. K. (1998). Auditory hallucinations: a review of psychological treatments. Schizophr. Res. 32, 137-150. doi: 10. 1016/s0920-9964(98)00052-8

Slotema, C. W., Aleman, A., Daskalakis, Z. J., and Sommer, I. E. (2012). Metaanalysis of repetitive transcranial magnetic stimulation in the treatment of auditory verbal hallucinations: update and effects after one month. Schizophr. Res. 142, 40-45. doi: 10.1016/j.schres.2012.08.025

Slotema, C. W., Blom, J. D., de Weijer, A. D., Diederen, K. M., Goekoop, R., Looijestijn, J., et al. (2011). Can low-frequency repetitive transcranial magnetic stimulation really relieve medication-resistant auditory verbal hallucinations? Negative results from a large randomized controlled trial. Biol. Psychiatry. 69, 450-456. doi: 10.1016/j.biopsych.2010.09.051

Slotema, C. W., Blom, J. D., Hoek, H. W., and Sommer, I. E. (2010). Should we expand the toolbox of psychiatric treatment methods to include Repetitive Transcranial Magnetic Stimulation (rTMS)? A meta-analysis of the efficacy of rTMS in psychiatric disorders. J. Clin. Psychiatry. 71, 873-884. doi: 10.4088/jcp. 08m04872gre

Slotema, C. W., Blom, J. D., van Lutterveld, R., Hoek, H. W., and Sommer, I. E. (2014). Review of the efficacy of transcranial magnetic stimulation for auditory verbal hallucinations. Biol. Psychiatry. 76, 101-110. doi: 10.1016/j.biopsych. 2013.09 .038

Sommer, I. E., de Weijer, A. D., Daalman, K., Neggers, S. F., Somers, M., Kahn, R. S., et al. (2007). Can fMRI-guidance improve the efficacy of rTMS treatment for auditory verbal hallucinations? Schizophr. Res. 93, 406-408. doi: 10.1016/j. schres.2007.03.020

Sommer, I. E., Diederen, K. M., Blom, J. D., Willems, A., Kushan, L., Slotema, K., et al. (2008). Auditory verbal hallucinations predominantly activate the right inferior frontal area. Brain. 131, 3169-3177. doi: 10.1093/brain/awn251 
Sommer, I. E., Slotema, C. W., Daskalakis, Z. J., Derks, E. M., Blom, J. D., and van der Gaag, M. (2012). The treatment of hallucinations in schizophrenia spectrum disorders. Schizophr. Bull. 38, 704-714. doi: 10.1093/schbul/sbs034

Stephane, M., Barton, S., and Boutros, N. N. (2001). Auditory verbal hallucinations and dysfunction of the neural substrates of speech. Schizophr. Res. 50, 61-78. doi: 10.1016/s0920-9964(00)00150-x

Tharyan, P., and Adams, C. E. (2005). Electroconvulsive therapy for schizophrenia. Cochrane Database Syst. Rev. 18:CD000076. doi: 10.1002/9780470987353.ch27

Thiel, A., Schumacher, B., Wienhard, K., Gairing, S., Kracht, L. W., Wagner, R., et al. (2006). Direct demonstration of transcallosal disinhibition in language networks. J. Cereb. Blood Flow Metab. 26, 1122-1127. doi: 10.1038/sj.jcbfm. 9600350

Tranulis, C., Sepehry, A. A., Galinowski, A., and Stip, E. (2008). Should we treat auditory hallucinations with repetitive transcranial magnetic stimulation? A metaanalysis. Can. J. Psychiatry. 53, 577-586.

Vlachos, A., Muller-Dahlhaus, F., Rosskopp, J., Lenz, M., Ziemann, U., and Deller, T. (2012). Repetitive magnetic stimulation induces functional and structural plasticity of excitatory postsynapses in mouse organotypic hippocampal slice cultures. J. Neurosci. 32, 17514-17523. doi: 10.1523/jneurosci.0409-12.2012

Wang, J. X., and Voss, J. L. (2015). Long-lasting enhancements of memory and hippocampal-cortical functional connectivity following multiple-day targeted noninvasive stimulation. Hippocampus. 25, 877-883. doi: 10.1002/hipo. 22416

Wassermann, E. M., and Zimmermann, T.. (2012). Transcranial magnetic brain stimulation: therapeutic promises and scientific gaps. Pharmacol. Ther. 133, 98-107. doi: 10.1016/j.pharmthera.2011.09.003
Waters, F., Allen, P., Aleman, A., Fernyhough, C., Woodward, T. S., Badcock, J. C., et al. (2012). Auditory hallucinations in schizophrenia and nonschizophrenia populations: a review and integrated model of cognitive mechanisms. Schizophr. Bull. 38, 683-693. doi: 10.1093/schbul/sbs045

Wolf, N. D., Grön, G., Sambataro, F., Vasic, N., Frasch, K., Schmid, M., et al. (2012). Magnetic resonance perfusion imaging of auditory verbal hallucinations in patients with schizophrenia. Schizophr. Res. 134, 285-287. doi: 10.1016/j.schres.2011.11.018

Wolf, N. D., Sambataro, F., Vasic, N., Frasch, K., Schmid, M., Schönfeldt-Lecuona, C., et al. (2011). Dysconnectivity of multiple resting-state networks in patients with schizophrenia who have persistent auditory verbal hallucinations. $J$. Psychiatry. Neurosci. 36, 366-374. doi: 10.1503/jpn.110008

Woodward, T. S., Jung, K., Hwang, H., Yin, J., Taylor, L., Menon, M., et al. (2014). Symptom dimensions of the psychotic symptom rating scales in psychosis: a multisite study. Schizophr. Bull. 4, S265-S274. doi: 10.1093/schbul/sbu014

Conflict of Interest Statement: The authors declare that the research was conducted in the absence of any commercial or financial relationships that could be construed as a potential conflict of interest.

Copyright (C) 2015 Kubera, Barth, Hirjak, Thomann and Wolf. This is an open-access article distributed under the terms of the Creative Commons Attribution License (CC $B Y)$. The use, distribution and reproduction in other forums is permitted, provided the original author(s) or licensor are credited and that the original publication in this journal is cited, in accordance with accepted academic practice. No use, distribution or reproduction is permitted which does not comply with these terms. 